\title{
Acrylate and methacrylate esters: Relationship of hemolytic activity and in vivo toxicity
}

\author{
E. O. Dillingham, W. H. Lawrence, and J. Autian \\ Materials Science Toxicology Laboratories, University of Tennessee Center for the Health \\ Sciences, Memphis, Tennessee 38163 \\ G. Schmalz \\ Eberhard-Karls Universitat Tubingen, Tubingen 1, West Germany
}

Quantitative hemolysis assays of acrylate and methacrylate esters provided estimates of the intrinsic hemolytic activity $\left(H_{i}\right.$, the slope of the concentration-response curve) and the concentrations effecting $5 \%\left(\mathrm{H}_{5}\right)$ and $50 \%\left(H_{50}\right)$ hemolysis. The dependence of hemolytic activity and $\mathrm{LD}_{50}$ (mice) on physical properties (lipophilicity, molar refraction, and molecular volume) of the esters was determined by multiple regression analysis. The observed correlations were: $H_{i}, R^{2}=0.94 ; H_{5}, R^{2}=0.95 ; H_{50}, R^{2}$ $=0.94 ;$ and $\mathrm{LD}_{50}, R^{2}$ (all compounds) $=0.80$, $R^{2}$ (all compounds less the methyl esters) = 0.94. The difference of the methyl esters was associated with the smaller steric vol- ume of the methyl ester substituent and the presence (methacrylates) or absence (acrylates) of the branched methyl group. Associative steric contributions of the branched methyl group and the ester substituents were probably responsible for greater variability in the methyacrylate series. The results were consistent with the conclusion that the mechanism of the action of the esters is membrane mediated and relatively nonspecific and that in vivo biotransformation was not a significant factor. Also, long-term toxic liability of the esters may be more closely related to intrinsic toxicity than acute toxicity.

\section{INTRODUCTION}

Acrylate and methacrylate esters are used widely in the formulation of polymeric materials for medical, dental, orthopedic, and industrial applications. The volatility of the esters make them a potential hazard in the work environment, and residual unpolymerized esters or esters released on degradation of acrylic or methacrylic polymers pose a toxic liability in the biological application of such materials. Although direct contact or inhalation poses the most serious hazard, more subtile questions of biological compatibility and potential toxic liability are present in in situ polymerization procedures contacting soft and hard tissues. In those procedures, tissues are exposed to significant concentrations of free monomer and the toxic liability of the esters is a primary concern.

Diechmann ${ }^{\mathrm{r}}$ and Spealman et al. ${ }^{2}$ tested a series of methacrylate esters in small animals and found the methyl ester to be the most toxic, followed by the ethyl and $n$-butyl esters. Pozzani, Weil, and Carpenter ${ }^{3}$ and Treon et al. ${ }^{4}$ reported that acute toxicity was correlated with water solubility; in vivo meth- 
ylacrylate was more toxic than ethylacrylate and both were more toxic than the corresponding methacrylate esters. Those conclusions were confirmed by Lawrence et al. ${ }^{5}$ and Bass et al. ${ }^{6}$ who examined the structure activity relationship of 18 acrylic and methacrylic compounds. Singh, Lawrence, and Autian ${ }^{7}$ reported presumptive evidence of teratogenicity of acrylates and methacrylates. The observed activity appeared to be directly correlated with water solubility. Mir, Lawrence, and Autian, 8,9 using isolated organ parameters, found no direct correlation of toxicity with water solubility except for the rate of contraction of rabbit heart.

Since in vivo biological activity depends simultaneously upon secondary time-dependent mechanisms (e.g., transport, biotransformation, and excretion) and intrinsic toxicity (the time-independent toxicant-receptor interaction), both of which are modulated by the physicochemical properties of the toxicant, the object of this investigation was to examine the relationship among those parameters directed toward rationalization of in vitro intrinsic toxicity with in vivo toxicity of acrylate and methacrylate esters.

\section{HEMOLYSIS ASSAY SYSTEM}

Evaluation of intrinsic biological activity requires an equilibrium system such as that described by Higuchi and Davis. ${ }^{10}$ Dillingham et al. ${ }^{11}$ demonstrated that a monolayer tissue culture assay meets the constraints described, and a high degree of correlation $(r=0.98)$ between tissue culture and hemolysis assays was reported for methyl-and halogen-substituted alcohols. The hemolysis assay system was selected over the tissue culture system based on the following considerations: (1) the opportunity for secondary hydrophobic partitioning to added serum is $60 \%$ lower, (2) oxidative metabolism in erythrocytes is low, (3) metabolic activity associated with growth and reproduction is absent, and (4) the assay time is short, $1 \mathrm{~h}$ as opposed to $72 \mathrm{~h}$ for the tissue culture system. The hemolysis assay system, therefore, more closely approximated an equilibrium system relatively unaffected by secondary timedependent processes.

\section{EXPERIMENTAL}

\section{Materials}

The acrylate and methacrylate esters (Table I) were used as received: compounds $1,2,7,8$, and $11 ;^{*}$ compounds 5 and $6 ; ;^{\dagger}$ and compounds $3,4,9$, and $10 . \ddagger$ The monoethylether of hydroquinone was present in concentrations of 5-15 ppm. Gas chromatography revealed no other significant quantities of organic compound. Additions in the hemolysis assay system of hydroquinone or the monoethylether of hydroquinone at concentrations two times higher did not change the apparent estimates of intrinsic hemolytic activity.

\footnotetext{
- Rohm and Haas, Philadelphia, PA.

Rohm and Haas, Deer Park, TX.

\$\&K Laboratories, Inc., Plainview, NY
} 


\section{Hemolysis assay}

Fresh oxalated rabbit blood ( $2 \%$ potassium oxalate in saline; $1 \mathrm{~mL} / 20 \mathrm{~mL}$ of rabbit blood) was diluted with normal saline such that $0.2 \mathrm{~mL}$ of diluted blood hemolyzed in $10 \mathrm{~mL}$ of distilled water gave a spectrophotometric reading at $545 \mathrm{~nm}$ of $0.9-1.0$ absorbance (positive control, $100 \%$ lysis).

Arbitrary concentrations near saturation of the esters in saline were employed as stock solutions. Preliminary range finding assays were carried out to establish the hemolysis concentration-response range and ten-step dilution series were prepared yielding hemolysis values between 2 and $95 \%$. The assays were carried out in test tubes with $10 \mathrm{~mL}$ in each tube and threefold replication at each concentration, including a negative control, normal saline. The tubes containing toxicant and control solutions were equilibrated for $30 \mathrm{~min}$ at $37^{\circ} \mathrm{C}$, and $0.20 \mathrm{~mL}$ of diluted rabbit blood was added to each tube with gentle mixing. After $60 \mathrm{~min}$ incubation following the addition of blood, all tubes were centrifuged for $5 \mathrm{~min}(500 \times g)$, the optical density (OD) of each supernatant was determined at $545 \mathrm{~nm}$, and replicate values averaged. The percent hemolysis was calculated as follows:

$$
\% \mathrm{H}=\frac{\mathrm{OD} \text { test solution }-\mathrm{OD} \text { negative control }}{\mathrm{OD} \text { positive control }-\mathrm{OD} \text { negative control }} \times 100
$$

Concentration-response curves were prepared and the biological response data $-H_{5}, H_{50}$, and $H_{i}$ (Table I) - were determined from smooth curves showing best fit with the mean values (visual estimation) (Fig. 1). The $\mathrm{H}_{5}$ and $H_{50}$ values were taken from the smooth curves. The slope of the concentration-response curve, $H_{i}$, was taken as the tangent to the curve at inflection point of the curve, the curves being relatively linear in that region, between 40 and $60 \%$ hemolysis.

TABLE I

Biological Response Parameters

\begin{tabular}{lcccc}
\hline \multicolumn{1}{c}{ Compound } & $\begin{array}{c}\mathrm{H}_{5} \\
(\mathrm{~mole} / \mathrm{L})^{\mathrm{a}}\end{array}$ & $\begin{array}{c}\mathrm{H}_{50} \\
(\mathrm{~mole} / \mathrm{L})^{\mathrm{b}}\end{array}$ & $\begin{array}{c}\mathrm{H}_{i} \\
(\% / \mathrm{mole})^{\mathrm{c}}\end{array}$ & $\begin{array}{c}\mathrm{LD}_{50} \\
\left(\mathrm{~mole} / 10^{6} \mathrm{~g}\right)^{\mathrm{d}}\end{array}$ \\
\hline 1. Methyl acrylate & $2.12 \times 10^{-1}$ & $2.33 \times 10^{-1}$ & $2.32 \times 10^{3}$ & 2.95 \\
2. Ethyl acrylate & $1.04 \times 10^{-1}$ & $1.11 \times 10^{-1}$ & $6.69 \times 10^{3}$ & 5.98 \\
3. $n$-Propyl acrylate & $1.86 \times 10^{-2}$ & $2.59 \times 10^{-2}$ & $2.33 \times 10^{4}$ & 5.80 \\
4. -Propyl acrylate & $1.38 \times 10^{-2}$ & $1.83 \times 10^{-2}$ & $2.79 \times 10^{4}$ & 6.42 \\
5. -Butyl acrylate & $1.48 \times 10^{-3}$ & $2.45 \times 10^{-3}$ & $1.20 \times 10^{5}$ & 6.64 \\
6. -Butyl acrylate & $9.65 \times 10^{-4}$ & $1.44 \times 10^{-3}$ & $3.46 \times 10^{5}$ & 5.92 \\
7. Methyl methacrylate & $7.15 \times 10^{-2}$ & $8.84 \times 10^{-2}$ & $8.87 \times 10^{3}$ & 11.29 \\
8. Ethyl methacrylate & $2.58 \times 10^{-2}$ & $3.60 \times 10^{-2}$ & $1.82 \times 10^{4}$ & 10.88 \\
9. n-Propyl methacrylate & $4.52 \times 10^{-3}$ & $6.83 \times 10^{-3}$ & $9.11 \times 10^{4}$ & 7.89 \\
10. -Propyl methacrylate & $2.47 \times 10^{-3}$ & $3.16 \times 10^{-3}$ & $2.28 \times 10^{5}$ & 11.63 \\
11. -Butyl methacrylate & $2.77 \times 10^{-4}$ & $3.83 \times 10^{-4}$ & $8.76 \times 10^{5}$ & 10.47 \\
\hline
\end{tabular}

a $H_{5}=$ concentration effecting $5 \%$ hemolysis.

b $H_{50}=$ concentration effecting $50 \%$ hemolysis.

c $H_{i}=$ slope of the dose-response curve between 40 and $60 \%$ hemolysis.

d Male ICR mice. 


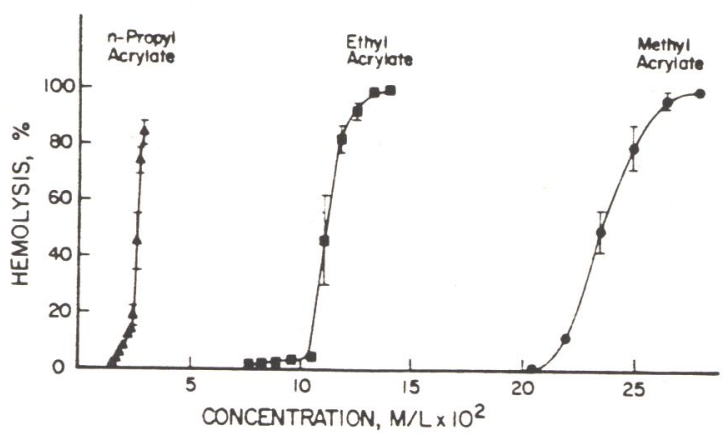

Figure 1. Hemolytic activity: typical concentration-response curves.

\section{In vivo toxicity}

The 7-day $\mathrm{LD}_{50}$ values (male ICR mice) used in this study were obtained from Lawrence et al. ${ }^{5}$ and Bass et al. ${ }^{6}$ (Table I).

\section{Physical parameters}

Physical parameters selected for correlation analysis with biological response included lipophilicity, molar refraction, and molecular volume (Table II). The $\log P$ values and the fragment constant values for calculating the lipophilicity of the $R_{1}$ and $R_{2}$ substituents of the acrylate and methacrylate esters were provided by Dr. Albert Leo, Pomona College, Claremont, California. Molar refraction and molecular volume values were obtained from the literature by Hansch and Leo ${ }^{12}$ and by Bondi, ${ }^{13}$ respectively.

\section{Correlation analysis}

Linear regression of biological parameters was carried out on all possible combinations of 1-5 physical parameters. The statistical program BMD P9R (ref. 14) was employed. The regression equation in each subset comprised of 1-4 independent variables (physical parameters) having the highest multiple regression coefficient $(R)$ was determined for the dependent variables $H_{i}, H_{5}$, $H_{50}$, and $L_{50}$. The addition of a fifth independent variable either did not improve or decreased $R$.

The correlation among physical parameters was examined by univariate correlation analysis.

\section{RESULTS AND DISCUSSION}

\section{Biological response parameters}

The biological response data are presented in Table I. Typical concentration-response curves are shown in Figure 1. The hemolytic activity of the acrylate and methacrylate esters increased with respect to the chain length 
TABLE II

Physical Parameters

\begin{tabular}{|c|c|c|c|c|c|c|c|c|c|c|}
\hline Compounda & $P$ & $\log P$ & $\pi \mathrm{R}_{1}$ & $\pi \mathrm{R}_{2}$ & MR & $\operatorname{MR}\left(R_{1}\right)$ & $\operatorname{MR}\left(R_{2}\right)$ & $V_{\omega}$ & $V_{\omega} R_{1}$ & $V_{\omega} R_{2}$ \\
\hline 1. Methyl acrylate & 4.217 & 0.625 & 0 & 0.89 & 21.85 & 0 & 5.65 & 49.02 & 3.44 & 13.67 \\
\hline 2. Ethyl acrylate & 14.622 & 1.165 & 0 & 1.55 & 26.03 & 0 & 10.30 & 59.25 & 3.44 & 23.90 \\
\hline 3. n-Propyl acrylate & 50.699 & 1.705 & 0 & 1.97 & 26.50 & 0 & 14.96 & 69.47 & 3.44 & 34.12 \\
\hline 4. i-Propyl acrylate & 30.549 & 1.485 & 0 & 1.97 & 26.50 & 0 & 14.96 & 69.47 & 3.44 & 34.12 \\
\hline 5. n-Butyl acrylate & 175.792 & 2.245 & 0 & 2.51 & 31.15 & 0 & 19.61 & 79.70 & 3.44 & 44.35 \\
\hline 6. i-Butyl acrylate & 272.270 & 2.435 & 0 & 2.51 & 31.15 & 0 & 19.61 & 79.70 & 3.44 & 44.35 \\
\hline 7. Methyl methacrylate & 8.810 & 0.945 & 0.89 & 0.89 & 27.50 & 5.65 & 5.65 & 59.25 & 13.67 & 13.67 \\
\hline 8. Ethyl methacrylate & 30.549 & 1.485 & 0.89 & 1.55 & 31.68 & 5.65 & 10.30 & 69.48 & 13.67 & 23.90 \\
\hline 9. n-Propyl methacrylate & 105.925 & 2.025 & 0.89 & 1.97 & 32.15 & 5.65 & 14.96 & 79.70 & 13.67 & 34.12 \\
\hline 10. $i$-Propyl methacrylate & 63.826 & 1.805 & 0.89 & 1.97 & 32.15 & 5.65 & 14.96 & 79.70 & 13.67 & 34.12 \\
\hline 11. n-Butyl methacrylate & 367.282 & 2.565 & 0.89 & 2.51 & 36.80 & 5.65 & 19.61 & 89.94 & 13.67 & 44.36 \\
\hline
\end{tabular}

$\mathrm{R}^{\prime} \quad \mathrm{O}$

a General structure: $\quad \mathrm{CH}=\mathrm{CH}-\mathrm{C}-\mathrm{O}-\mathrm{R}_{2}$,

where $R_{1}=$ hydrogen or methyl and $R_{2}=$ methyl, ethyl, propyl, isopropyl, butyl, or isobutyl. 


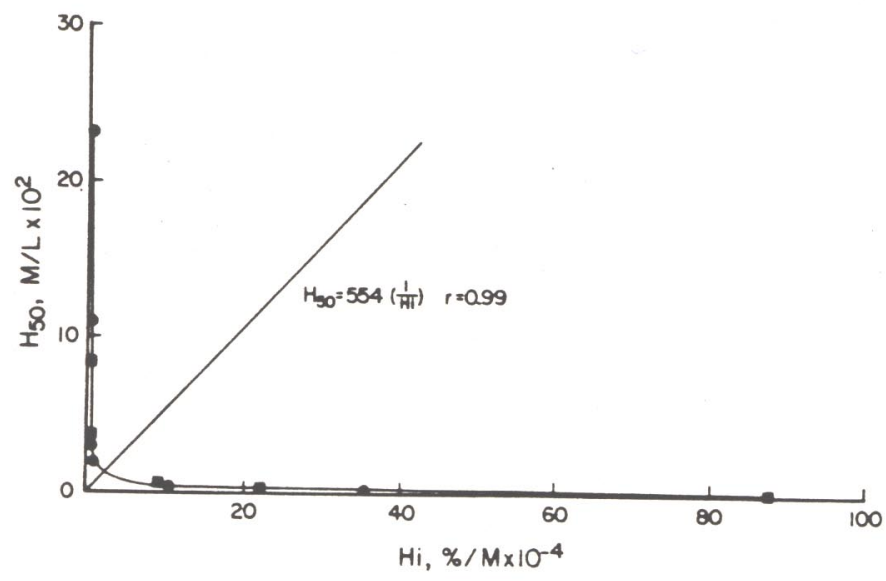

Figure 2. Correlation of $H_{50}$ and $H_{i}$. •, Acrylates; athacrylates.

of the ester substituent, with the methacrylate esters showing uniformly higher activity. All isocompounds had slightly lower activity compared to the corresponding straight chain compounds. The observed intrinsic hemolytic activity $\left(H_{i}\right)$ was inversely proportional to water solubility, agreeing more closely with isolated organ response than in vivo toxicity. ${ }^{1-9}$

The relationship between $H_{i}$ and $H_{50}$ (Fig. 2) is hyperbolic $(r=0.99)$, which is consistent with the conclusion that the hemolysis assay system is a pseudoequilibrium system appropriate for the estimation of intrinsic biological activity, ${ }^{10}$ in this case, intrinsic hemolytic activity. The same basic hyperbolic relationship was observed between $H_{i}$ and $H_{5}$. The slope of the concentration-response curve, therefore, was considered to be a valid estimate of intrinsic hemolytic activity $\left(H_{i}\right)$. The relationship between primary toxicantmembrane interaction, as expressed by hemolytic activity, and in vivo toxicity was examined through correlation analysis. Although correlation per se does not establish mechanism, it is influenced by mechanism and is relevant to the assessment of in vitro assay systems for prediction of in vivo toxicity.

TABLE III

Squared Correlation Matrix: Physical Parametersa

\begin{tabular}{llllllllll}
\hline & $\log P$ & $\pi \mathrm{R}_{1}$ & $\pi \mathrm{R}_{2}$ & $\mathrm{MR}$ & $\mathrm{MR}\left(\mathrm{R}_{1}\right)$ & $\mathrm{MR}\left(\mathrm{R}_{2}\right)$ & $V_{\omega}$ & $V_{\omega} \mathrm{R}_{1}$ & $V_{\omega} \mathrm{R}_{2}$ \\
\hline $\log \mathrm{P}$ & $1.00^{\mathrm{b}}$ & & & & & & & & \\
$\pi \mathrm{R}_{1}$ & 0.02 & 1.00 & & & & & & & \\
$\pi \mathrm{R}_{2}$ & 0.91 & 0.01 & 1.00 & & & & & & \\
$\mathrm{MR}$ & 0.70 & 0.38 & 0.48 & 1.00 & & & & \\
$\mathrm{MR}\left(\mathrm{R}_{1}\right)$ & 0.02 & 1.00 & 0.01 & 0.38 & 1.00 & & & \\
$\mathrm{MR}\left(\mathrm{R}_{2}\right)$ & 0.91 & 0.01 & 0.99 & 0.46 & 0.01 & 1.00 & & \\
$V_{\omega}$ & 0.92 & 0.12 & 0.80 & 0.84 & 0.12 & 0.80 & 1.00 & & \\
$V \omega R_{1}$ & 0.02 & 1.00 & 0.01 & 0.38 & 1.00 & 0.01 & 0.12 & 1.00 & \\
$V \omega \mathrm{R}_{2}$ & 0.91 & 0.01 & 0.99 & 0.46 & 0.01 & 1.00 & 0.80 & 0.01 & 1.00 \\
\hline
\end{tabular}

a See Table II.

b Matrix values are univariate correlation coefficients squared. 
TABLE IV

Linear Regression Analysis: Biological Response on Physical Parameters

\begin{tabular}{|c|c|c|c|c|c|c|}
\hline \multirow{2}{*}{\multicolumn{2}{|c|}{$\begin{array}{l}\text { Log biological } \\
\text { response }\end{array}$}} & \multicolumn{5}{|c|}{ Statistics $n=11$} \\
\hline & & $R^{2}$ & $d f^{\mathrm{a}}$ & $S^{b}$ & $F$ & $\begin{array}{l}\text { Equation } \\
\text { number }\end{array}$ \\
\hline \multirow[t]{4}{*}{$H_{t}$} & $=1.23 \log P+2.59$ & 0.900 & 1,9 & 0.265 & 81.1 & 1 \\
\hline & $=0.11 \mathrm{MR}\left(\mathrm{R}_{1}\right)+0.07 V_{\omega} R_{2}+2.31$ & 0.944 & 2,8 & 0.210 & 67.7 & 2 \\
\hline & $=0.241 \log P+0.09 \mathrm{MR}+0.03 V_{\omega} \mathrm{R}_{2}+0.76$ & 0.933 & 3,7 & 0.246 & 32.4 & 3 \\
\hline & $=0.371 \log P-0.008 \mathrm{MR}+0.10 \mathrm{MR}\left(\mathrm{R}_{1}\right)+0.05 V_{\omega} \mathrm{R}_{2}+2.5$ & 0.946 & 4,6 & 0.238 & 26.4 & 4 \\
\hline \multirow[t]{4}{*}{$\mathrm{H}_{5}$} & $=1.44 \log P-0.39$ & 0.931 & 1,9 & 0.254 & 122.2 & 5 \\
\hline & $=0.10 \mathrm{MR}+0.05 V_{\omega} \mathrm{R}_{2}-2.50$ & 0.949 & 2,8 & 0.232 & 74.8 & 6 \\
\hline & $=0.05 \mathrm{MR}+0.06 \mathrm{MR}\left(\mathrm{R}_{1}\right)+0.06 V_{\omega} \mathrm{R}_{2}-1.54$ & 0.952 & 3,7 & 0.239 & 47.1 & 7 \\
\hline & $=0.42 \log P+0.02 \mathrm{MR}+0.06 \mathrm{MR}\left(\mathrm{R}_{1}\right)+0.05 V_{\omega} \mathrm{R}_{2}-1.00$ & 0.954 & 4,6 & 0.253 & 31.5 & 8 \\
\hline \multirow[t]{4}{*}{$H_{50}$} & $=1.36 \log P-1.39$ & 0.920 & 1,9 & 0.261 & 103.6 & 9 \\
\hline & $=0.10 \mathrm{MR}+0.05 V_{\omega} \mathrm{R}_{2}-2.40$ & 0.941 & 2,8 & 0.235 & 64.9 & 10 \\
\hline & $=0.05 \mathrm{MR}+0.05 \mathrm{MR}\left(\mathrm{R}_{1}\right)+0.06 V_{\omega} \mathrm{R}_{2}-1.52$ & 0.945 & 3,7 & 0.244 & 40.3 & 11 \\
\hline & $=0.27 \log P+0.03 \mathrm{MR}+0.06 \mathrm{MR}\left(\mathrm{R}_{1}\right)+0.05 V_{\omega} \mathrm{R}_{2}-1.17$ & 0.946 & 4,6 & 0.262 & $26.3_{\omega}$ & 12 \\
\hline & 0.666 & 1,9 & 0.108 & 17.9 & 13 \\
\hline \multicolumn{2}{|c|}{$\begin{aligned} L_{50} & =-0.05 \mathrm{MK}\left(\mathrm{K}_{1}\right)+2.26 \\
& =0.25 \log P-0.06 \mathrm{MR}+3.57\end{aligned}$} & 0.756 & 2,8 & 0.098 & 12.4 & 14 \\
\hline \multicolumn{2}{|r|}{$=0.30 \log P-0.40 \pi \mathrm{R}_{2}-0.07 \mathrm{MR}\left(\mathrm{R}_{1}\right)+2.54$} & 0.783 & 3,7 & 0.098 & 8.46 & 15 \\
\hline & $=0.391 \log P-0.33 \pi \mathrm{R}_{2}-0.03 \mathrm{MR}-0.04 \mathrm{MR}\left(\mathrm{R}_{1}\right)+3.07$ & 0.800 & 4,6 & 0.102 & 6.01 & 16 \\
\hline
\end{tabular}

- Degrees of freedom: numerator and denominator.

b Standard error of estimates. 


\section{Physical parameters}

Three basic physical parameters were used in the analysis: $P$ (octanol/water partition coefficient), $V \omega$ (molecular volume, $\mathrm{cm}^{3} / \mathrm{mole}$ ), and MR (molar refraction). The first two have been widely used in correlation analysis, while MR has been used relatively recently. ${ }^{12}$ Values for the parent compound and for the $R_{1}$ and $R_{2}$ substituents (Table II) were employed in the analysis.

The cross-correlation of physical parameters was examined by univariate correlation analysis (Table III). Where significant cross-correlations were present, the parameter giving the highest multiple correlation with biological response was selected.

\section{Correlation analysis}

Regression of biological response $\left(H_{i}, H_{5}, H_{50}\right.$, and $\left.\mathrm{LD}_{50}\right)$ on all possible subsets of physical parameters (Table II) was executed using statistical program BMD P9R. ${ }^{14}$ The best one-through four-parameter equations are found in Table IV. The four-parameter equations uniformly gave the highest multiple correlation $\left(R^{2}\right)$ with biological response [eqs. (4), (8), (12), and (16)]. However, the two-parameter equations [eqs. (2), (6), and (10)] with independent variables MR or $M R\left(R_{1}\right)$ and $V_{\omega} R_{2}$ provide almost as good correlation as the four-parameter equations. The improvement of $R^{2}$ on the addition of $V_{\omega} R_{2}$ is statistically significant but small in magnitude compared to the correlation with the best single parameter, $\log P$ [eqs. (1), (5), and (9)]. The relationship of $H_{50}$ and $\log P$ is shown in Figure 3. The relationship is very similar for $\mathrm{H}_{5}$ and $H_{i}$ except that isobutyl acrylate showed slightly lower activity falling off the trend for the corresponding straight chain compounds.

Although the equations generated are the best selected by the statistical program employed, they are not necessarily better than equations that substitute highly cross-correlated parameters for the parameter appearing in the selected equation (Table IV). The $\mathrm{R}_{1}$ parameters only reflect the presence or absence of the methyl group and any one functions equally well as an indicator

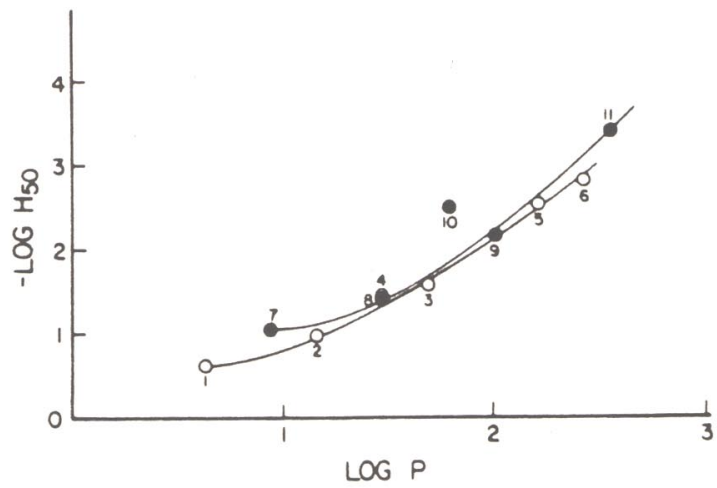

Figure 3. Relationship of $H_{50}$ and $\log P$. See Table I for compound identification. O, Acrylates; methacrylates. 


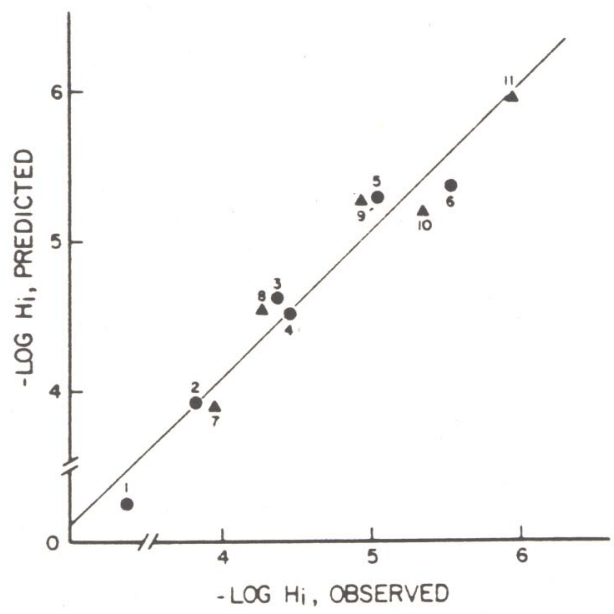

Figure 4. $H_{i}$ observed (Table I) and predicted [eq. (4), Table IV]. See Table I for compound identification. $\bullet$, Methacrylates; $\boldsymbol{\Lambda}$, acrylates.

variable in the analysis. This is particularly apparent in the equations for $\mathrm{LD}_{50}$ [eqs. (13)-(16)]. Any of the $R_{1}$ parameters discriminate $L_{50}$ equally well in the one-parameter equation, a reflection of the fact that $\mathrm{LD}_{50}$ values for the acrylate and methacrylate esters are significantly different in magnitude and show no well-defined trends with respect to the ester substituents. The overall correlation of $\mathrm{LD}_{50}$ with physical parameters is lower than similar correlations with hemolytic activity. A comparison of observed and predicted values for hemolysis and $L D_{50}$ data are found in Figures 4-7. For the $L_{50}$, it is apparent that the methyl esters are outliers that contribute significantly to the reduction of the overall correlation (univariate correlation of observed and predicted values) $r$ (overall) $=0.89$ and $r$ (all compounds less the methyl esters $)=0.97$,

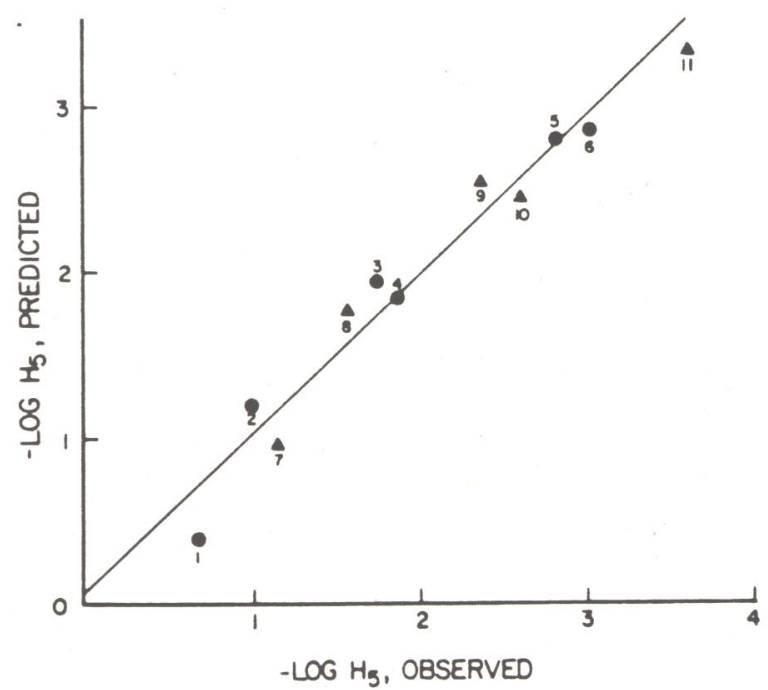

Figure 5. $H_{5}$ observed (Table I) and predicted [eq. (8), Table IV]. See Table I for compound identification. Acrylates; $\Delta$, methacrylates. 


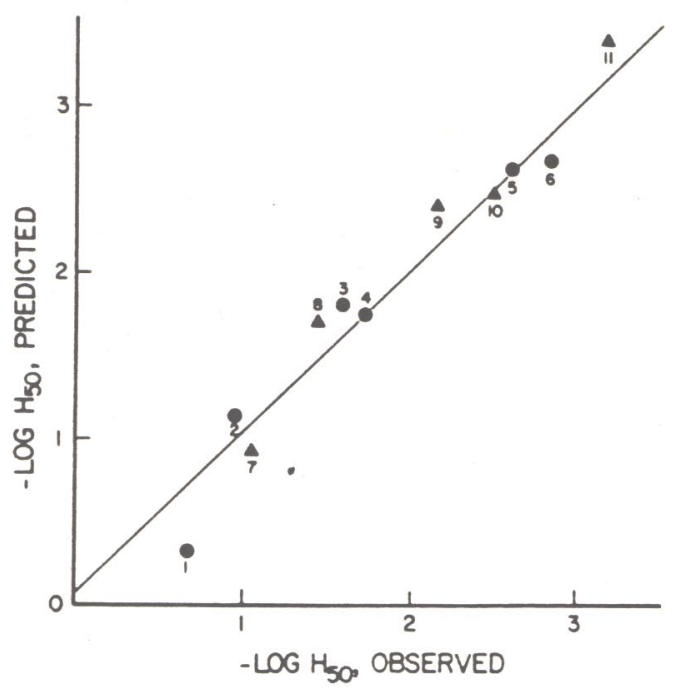

Figure 6. $H_{50}$ observed (Table I) and predicted [eq. (12), Table IV]. See Table I for compound identification. • Acrylates; $\mathbf{\Lambda}$, methacrylates.

the latter equal to the correlation with in vitro data. Since the predicted value for methyl methacrylate is high and for methyl acrylate is low, the steric parameters of both the $R_{1}$ and $R_{2}$ substituents are probably important to the differential expression of toxicity of those compounds in vivo. The relatively good correlations for all compounds where $R_{2}$ is ethyl or larger suggests that $\mathrm{R}_{2}$ is a primary factor in the expression of toxicity. The associative contributions of $R_{1}$ and $R_{2}$, however, are probably responsible for the greater variability within the methacrylate esters.

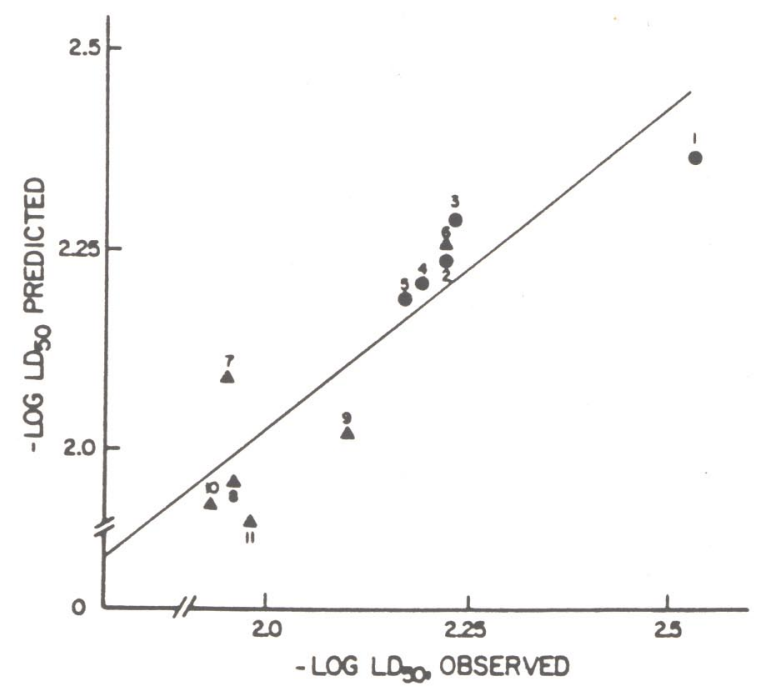

Figure 7. $L_{50}$ observed (Table I) and predicted [eq. (16), Table IV]. See Table I for compound identification. $\bullet$, Acrylates; $\boldsymbol{\Lambda}$, methacrylates. 


\section{Relationship of in vitro and in vivo response}

The excellent correlation of biological response with physical parameters and the uniformity of physical parameters in the four-parameter equations, $\pi \mathrm{R}_{2}$ being highly cross-correlated with $V_{\omega} \mathrm{R}_{2}$, suggests a fundamental relationship between the in vitro and in vivo biological response. The direct correlations (ratios) of in vitro response $\left(H_{i}\right)$ to in vivo response $\left(\mathrm{LD}_{50}\right)(A$, Table $\mathrm{V})$ show a very large (106-fold) variation. The correlation analysis indicates $\log P$ to be a dominant factor modulating biological response. To the degree that the reciprocal of the octanol/water partition coefficient $(1 / P)$ is a valid estimate of the effective aqueous concentration of low molecular weight toxicants in vivo, and to the degree that the intrinsic biological activity of toxicants is the same in vitro and in vivo, the product of $A$ and $1 / P(B$, Table $\mathrm{V})$ should be a constant if biotransformation is not a significant factor. The overall variation of $B$ is 5.6-fold compared to 106 -fold for $A$. Since the largest variation was in the methacrylate group, those values apply also to that group. For the acrylate group, the value of $A$ was 78 -fold and the value of $B$ was 2.8 -fold. The differential response $(B)$ of methyl acrylate and methyl methacrylate is apparent as is the greater variability of $B$ for the members of the methacrylate group, probably modulated by the associative steric relationships of $R_{1}$ and $\mathrm{R}_{2}$. Since it can reasonably be assumed that intrinsic biological activity $\left(H_{i}\right)$ can be free of secondary processes of biotransformation, transport and excretion, the residual variation in $B$ may be related to those in vivo processes. It is not possible, however, to discriminate among those factors and statistical error in the present analysis. The overall variation of $B(5.6$-fold) is approximately the same as observed previously for methyl-and halogen-substituted alcohols $^{11}$ where similar relationships with lipophilicity and steric parameters were demonstrated. Biotransformation of a toxicant to significantly higher or lower toxicity metabolites can be expected to significantly affect the $L_{50}$ and the value of $B$, as demonstrated in the alcohol study. ${ }^{11}$ Since the overall

TABLE V

Relationship of $P$ to in vitro-in vivo Response

\begin{tabular}{lcc} 
Compound & $\begin{array}{c}A=H_{i} / \mathrm{LD}_{50} \\
\times 10^{-5}\end{array}$ & $\begin{array}{c}B=H_{i} / \mathrm{LD}_{50} P \\
\times 10^{-5}\end{array}$ \\
\hline 1. Methyl acrylate & 7.9 & 1.87 \\
2. Ethyl acrylate & 11.2 & 0.77 \\
3. $n$-Propyl acrylate & 40.2 & 0.79 \\
4. $i$-Propyl acrylate & 43.4 & 1.42 \\
5. $n$-Butyl acrylate & 180.6 & 1.03 \\
6. $i$-Butyl acrylate & 584.0 & 2.15 \\
7. Methyl methacrylate & 7.9 & 0.89 \\
8. Ethyl methacrylate & 16.73 & 0.55 \\
9. $n$-Propyl methacrylate & 115.5 & 1.09 \\
10. $i$-Propyl methacrylate & 196.0 & 3.07 \\
11. $n$-Butyl methacrylate & 837.0 & 2.28 \\
Variation: & & \\
\hline
\end{tabular}


variability of the acrylate and methacrylate esters with respect to $B$ was of the same order of magnitude as that of the alcohols, the observed variability is consistent with the conclusion that the metabolites of those compounds in the course of a 7-day $\mathrm{LD}_{50}$ assay, where there is ample time for biotransformation, do not significantly affect the $\mathrm{LD}_{50}$, i.e. the compounds act by the same basic mechanism modulated by the physicochemical properties of the parent compound.

That in vivo toxicity in mice can be rationalized to a significant degree through consideration of in vitro intrinsic hemolytic activity supports the conclusion that the mechanism of action is membrane mediated and relatively nonspecific for acrylate and methacrylate esters. Intrinsic toxicity is related to lipophilicity (inversely related to water solubility), which suggests that the toxic liability of long-term exposure in vivo may be more closely related to intrinsic toxicity than acute toxicity which is consistent with observed differences in acute and chronic $L_{50}$ values for the phthalate esters. ${ }^{15}$ The value of in vitro assay systems for the estimation of intrinsic biological activity and the relevance of intrinsic activity to higher order biological response were demonstrated. 16

\section{References}

1. W. Deichmann, "Toxicity of Methyl, Ethyl and N-Butyl Methacrylate," J. Ind. Hyg. Toxicol., 23, 1343-1351 (1941).

2. C. R. Spealman, R. J. Main, H. B. Haag, and P. S. Larson, "Monomer Methyl Methacrylate-Studies on Toxicity," Ind. Med., 14, 292-298 (1945).

3. V. E. Pozzani, C. S. Weil, and C. P. Carpenter, "Subacute Vapor Toxicity and Range-finding Data for Ethyl Acrylate," I. Ind. Hyg. Toxicol., 31, 311-316 (1949).

4. J. F. Treon, H. Sigmon, H. Wright, and K. V. Kitzmiller, "The Toxicity of Methyl and Ethyl Acrylate," I. Ind. Hyg. Toxicol., 31, 317-326 (1949).

5. W. H. Lawrence, G. E. Bass, W. P. Purcell, and J. Autian, "Use of Mathematical Models in the Study of Structure-Toxicity Relationships of Dental Compounds. I. Esters of Acrylic and Methacrylic Acids," J. Dent. Res., 51, 526-535 (1972).

6. G. E. Bass, W. H. Lawrence, W. P. Purcell, and J. Autian, "Further Evaluation of a Quantitative Mathematical Model for Predicting Acute Toxicity of Acrylate and Methacrylate Esters," I. Dent. Res., 51, 1632-1638 (1972).

7. A. R. Singh, W. H. Lawrence, and J. Autian, "Embryonic-Fetal Toxicity and Teratogenic Effects of a Group of Methacrylate Esters in Rats," J. Dent. Res., 51, 1632-1638 (1972).

8. G. N. Mir, W. H. Lawrence, and J. Autian, "Toxicological and Pharmacological Actions of Methacrylate Monomers. I. Effects on Isolated, Perfused Rabbit Heart," J. Pharm. Sci., 62, 778-782 (1973).

9. G. N. Mir, W. H. Lawrence, and J. Autian, "Toxicological and Pharmacological Actions of Methacrylate Monomers. II. Effects on Isolated Guinea Pig Ileum," J. Pharm. Sci., 62, 1258-1261 (1973).

10. T. Higuchi and S. S. Davis, "Thermodynamic Analysis of Structure-Activity Relationships of Drugs: Prediction of Optimal Structure," I. Pharm. Sci., 59, 1376-1383 (1970).

11. E. O. Dillingham, R. W. Mast, G. E. Bass, and J. Autian, "Toxicity of Methyland Halogen-Substituted Alcohols in Tissue Culture Relative to Structure Activity Models and Acute Toxicity in Mice," I. Pharm. Sci., 62, 22-30 (1973). 
12. C. H. Hansch and A. Leo, Substituent Constants for Correlation Analysis in Biology and Chemistry, Wiley, New York, 1979.

13. A. Bondi, "van der Waals Volumes and Radii," J. Phys. Chem., 68, 441-450 (1964).

14. Statistical Program BMD, $P 9 R$, developed at the University of California Los Angeles, California, sponsored by NIH Special Resources Grant RR-3, revised Dec. 1979.

15. W. H. Lawrence, M. Malik, J. E. Turner, A. R. Singh, and J. Autian, "A Toxicological Investigation of Some Acute Short-Term and Chronic Effects of Administering Di-2-ethylhexyl Phthalate and Other Phthalate Esters," Environ. Res., 9, 1-11 (1975).

16. E. O. Dillingham, G. Schmalz, W. H. Lawrence, and J. Autian, "Acrylate and Methacrylate Esters: In Vitro-In Vivo Toxicity Relationships," presented at the Eighth Annual Meeting of the Society for Biomaterials Symposium, Orlando, FL, Apr. 24-27, 1982.

Received June 25, 1982

Accepted March 2, 1983 